\title{
Corrigendum: Shock Index, Pediatric Age-Adjusted Predicts Morbidity and Mortality in Children Admitted to the Intensive Care Unit
}

\author{
Kuo-Chen Huang ${ }^{1 \dagger}$, Ying Yang ${ }^{1 \dagger}$, Chao-Jui Li ${ }^{1}$, Fu-Jen Cheng ${ }^{1}$, Ying-Hsien Huang ${ }^{2}$, \\ Po-Chun Chuang ${ }^{1 *}$ and I-Min Chiu ${ }^{1 *}$ \\ ${ }^{1}$ Department of Emergency Medicine, Kaohsiung Chang Gung Memorial Hospital, Kaohsiung, Taiwan, ${ }^{2}$ Department of \\ Pediatrics, Kaohsiung Chang Gung Memorial Hospital, Kaohsiung, Taiwan
}

Keywords: pediatric, SIPA, shock index, mortality, emergency department, intensive care unit

\section{A Corrigendum on}

Shock Index, Pediatric Age-Adjusted Predicts Morbidity and Mortality in Children Admitted to the Intensive Care Unit

by Huang, K.-C., Yang, Y., Li, C.-J., Cheng, F.-J., Huang, Y.-H., Chuang, P.-C., and Chiu, I.-M. (2021). Front. Pediatr. 9:727466. doi: 10.3389/fped.2021.727466

OPEN ACCESS

Approved by:

Frontiers Editorial Office,

Frontiers Media SA, Switzerland

*Correspondence:

Po-Chun Chuang

zhungboqun@gmail.com

I-Min Chiu

outofray@hotmail.com

†These authors have contributed equally to this work

Specialty section

This article was submitted to Pediatric Critical Care, a section of the journal Frontiers in Pediatrics

Received: 01 December 2021 Accepted: 02 December 2021 Published: 21 December 2021

Citation:

Huang K-C, Yang Y, Li C-J, Cheng F-J, Huang $Y-H$, Chuang P-C and Chiu I-M (2021) Corrigendum:

Shock Index, Pediatric Age-Adjusted Predicts Morbidity and Mortality in Children Admitted to the Intensive Care Unit. Front. Pediatr. 9:827191. doi: 10.3389/fped.2021.827191
In the original article, there was an error. The date of approval of the study was incorrectly listed as "May 15, 2020." The study was approved by the Institutional Review Board of the Chang Gung Medical Foundation on May 15, 2021.

A correction has been made to Materials and Methods, Paragraph 1:

"This retrospective observational study was conducted at the EDs of three medical institutes (the Linkou, Chiayi, and Kaohsiung branches) located in northern and southern Taiwan. There were $35,000,11,000$, and 30,000 annual pediatric ED visits, respectively. The study was approved by the Institutional Review Board of the Chang Gung Medical Foundation (date of approval: May 15, 2021, number: 202100692B0). Patient and physician records and information were collected from the research database in the studied medical foundation and anonymized prior to the analysis."

A correction has been made to Ethics Statement:

"The study was approved by the Institutional Review Board of the Chang Gung Medical Foundation (date of approval: May 15, 2021, number: 202100692B0). Patient and physician records and information were collected from the research database in the studied medical foundation and anonymized prior to the analysis."

The authors apologize for this error and state that this does not change the scientific conclusions of the article in any way. The original article has been updated.

Publisher's Note: All claims expressed in this article are solely those of the authors and do not necessarily represent those of their affiliated organizations, or those of the publisher, the editors and the reviewers. Any product that may be evaluated in this article, or claim that may be made by its manufacturer, is not guaranteed or endorsed by the publisher.

Copyright () 2021 Huang, Yang, Li, Cheng, Huang, Chuang and Chiu. This is an open-access article distributed under the terms of the Creative Commons Attribution License (CC BY). The use, distribution or reproduction in other forums is permitted, provided the original author(s) and the copyright owner(s) are credited and that the original publication in this journal is cited, in accordance with accepted academic practice. No use, distribution or reproduction is permitted which does not comply with these terms. 